\title{
Entrar y sentarse en el ombligo del mundo / o trazos zen en la poética de Hugo Padeletti
}

\section{Entering and sitting at the navel of the world /or Zen strokes in Hugo Padeletti's poetic}

Tania Favela Bustillo Universidad Iberoamericana, México Tania.favela@ibero.mx

Resumen: En el presente artículo me propongo señalar las relaciones entre la poética de Hugo Padeletti (Alcorta, Argentina,I928-2018) y el budismo zen, tomando en cuenta el arte del té y el zazen (concentración sedante de la escuela Soto). El eje de la discusión se centrará en dos acciones simples que conllevan una concepción del mundo y del hombre: entrar en un recinto y sentarse. ¿Qué implican estas dos acciones desde la perspectiva del budismo zen? y ¿qué sentido tienen en la poética padelettiana? Seguir algunos trazos de la "contemplación serena" y de la estética zen, permitirá mostrar una parte del recorrido que Padeletti emprendió, con intuición e inteligencia, hacia lo que él llamó su camino laico de realización interior.

Palabras claves: Poesía, zen, intuición, Argentina.

Abstract: In the present article my proposal is to point out the relation between Hugo Padeletti's (Alcorta, Argentina, 1928- 2018) poetic and Zen Buddhism, taking in account the art of tea and zazen (Soto 
school of concentration by sitting down). The axis of the discussion will be centered in two simple actions that bring us to a conception of the world and human beings: entering a room and sitting down. What this two-actions imply in Zen Buddhism's perspective? And what is their purpose in the padelettian poetic? Following some traces of "serene contemplation" and Zen aesthetics, will allow to show a part of Padeletti's journey that with intuition and intelligence he undertook, towards what he called his secular way to interior realization.

Keywords: Poetry, Zen, intuition, Argentina.

Recibido: 20 de enero de $202 \mathrm{I}$ Aceptado: Io de abril de 202 I https://dx.doi.org/IO.I 5 I74/rv.vi3i28.598

Cuando regresamos a la realidad, la realidad no es un concepto.

Dogen

\begin{abstract}
Comienzo con una memoria de infancia que Hugo Padeletti Jevoca al recordar los recorridos que hacía con su padre por las chacras de la zona:
\end{abstract}

Una vez, en uno de esos viajes, llegamos a un cruce de tres calles o caminos en pleno campo. El cruce determinaba un triángulo de dos o tres metros de lado, cubierto de yuyos, cercado de alambre de púa y con un solo árbol copudo en el centro. Inmediatamente, quise bajar y meterme en ese recinto. Le pedí a mi padre que fuera hasta la chacra vecina y volviera después a buscarme. Mi padre dudó, pero me hizo pasar entre los alambres de púa y me 
dejó sentado bajo el árbol. Para él fue acceder a un capricho infantil; para mí a un encantamiento. No supe, hasta muchos ańos después, que había descubierto mi primer lugar sagrado: había encontrado, al mismo tiempo, el ombligo del mundo, el eje del mundo o el árbol de la vida, el paraíso terrenal que lo incluye y la técnica simple de la contemplación sentada (en japonés, zazen) que ya nunca, de uno u otro modo, me abandonó. Esta anécdota no parece venir al caso, pero ocurre que, cuando entro en estado de escribir poesía, dibujar, hacer collage, hacer objetos o pintar, siento, cómodamente, que estoy sentado en el centro (2004: 38).

El recuerdo de Padeletti nos lleva a pensar en una iniciación y también en un destino. Él mismo habla de sí como un seeker, un persistente buscador de sentido en el errante camino de esta vida. En su viaje por esta tierra, que duró casi 90 ańos, discurrió por el cristianismo, la teosofía, la filosofía (titulándose con una monografía sobre la estética de Heidegger), el hinduismo, el taoísmo, el budismo y el budismo zen. Se interesó por la astrología, el esoterismo y el tarot, e hizo suya la experiencia estético-metafísica a través de la poesía y de las artes plásticas. Aunque en su obra podemos encontrar las huellas de todos sus variados intereses, es, sin duda, el budismo zen el que mayor gravitación tuvo en su vida. El zazen, como práctica de la contemplación, está, como él mismo lo anota, en el origen de esa anécdota de infancia y lo acompañó hasta su vejez.

Hay dos momentos que quiero resaltar de esta memoria de infancia: "quise bajar y meterme en ese recinto" y "me dejó sentado bajo el árbol”. Entrar en el recinto y sentarse: ¿qué implican estas dos acciones desde la perspectiva del budismo zen?, ¿qué sentido tienen en la obra poética de Hugo Padeletti? Sentarse implica en el zen, el zazen: concentración sedante de la escuela Soto. Según Shituzeru Ueda, sentarse es, de las cuatro posturas básicas que existen 
para el budismo: caminar, estar en pie, sentarse y yacer, la postura principal del zen. La reflexión de Ueda al respecto es muy interesante:

En antropología, la postura erguida es considerada como la característica determinante de la existencia humana [...] Con la postura bípeda, nuestras manos quedaron libres, proporcionándonos la apertura necesaria para trascender nuestro entorno biológico [...] Por el contrario, el sentarse con las manos y las piernas inmóviles anula todos los medios de superioridad biológica del hombre y le devuelve, por un momento, al punto de "no hacer". Desde luego, esto implica una especie de actividad inversa: un hacer del no hacer (2005: 35-37).

Sentarse es para Ueda la postura de no confrontación: "El zazen es el modo de ser en que uno no se confronta con ninguna cosa; simplemente, se está abierto en una apertura infinita y como tal no se posee un yo" (2005: 41). En suma, sentarse es más que una postura corporal, es una postura ante la vida, una postura desjerarquizante, ya que al anular la postura erguida en la que el yo es el centro, se anula también la estructura antropocéntrica que impera en el mundo, y se abren otras formas de experimentarlo y de pensarlo; se abre el "más allá del pensamiento" o el "pensar sin pensar" (hishiryo), que sería, según el mismo Padeletti, un pensar desde la intuición y no desde la razón. Taisen Deshimaru, en su libro La práctica del Zen, explica el paso del cerebro intelectual al cerebro intuitivo: "En zazen, al principio pensamos con el cerebro frontal; luego, los pensamientos del prefrontal se agotan y la conciencia se ahonda, penetrando hasta el cerebro primitivo o rinocéfalo, y el córtex entra en fase de reposo. A continuación, podemos alcanzar el tálamo, el cerebro intuitivo" (2000: 141). Padeletti practicó por más de veinte años la "contemplación serena" de la escuela Soto 
del zen, pero más allá de esa disciplina espiritual, su práctica, como camino laico hacia la realización interior, se extendió a su vida cotidiana y a lo que en ella tenía mayor peso: su vocación artística: ${ }^{1}$

Mis 'ejercicios espirituales' [anota] son ahora casi exclusivamente mis collages, mis dibujos, mis pinturas, mis poemas [...] Pero así como en la escuela Soto no se busca un satori distinto de la práctica misma (el satori es 'sólo sentarse'), aquí el satori es sólo la obra: la concentración y contemplación compositivas, a veces agotadoras, y la gozosa contemplación final de la obra ya lograda (Padeletti, 1999a: 140).

Entrar y sentarse. En su relato de infancia, hacia el final, el poeta señala: "ocurre que, cuando entro en estado de escribir poesía [...] siento, cómodamente, que estoy sentado en el centro”. Siguiendo a Ueda, podría decirse que Padeletti entra al "estado de escribir" en una postura de "no confrontación", entra a una "apertura infinita" con su mente intuitiva. El centro para el poeta argentino no está ya en ese "recinto" exterior, sino adentro de sí mismo. Desde su primer libro, Apuntamientos en el Ashram (1944-1946), anota a pie de página: "encontré la palabra (Ashram) en una obra sobre la India y, sin saber gran cosa todavía de su significado, la convertí en el símbolo de ese espacio interior" (Padeletti, 2018a: 35). Entrar a "sí mismo" y "entrar al poema" supone la misma acción. El espacio

${ }^{1}$ En correo electrónico a Luis Verdejo (pintor y poeta mexicano), Padeletti comenta: "Veo que compartimos la misma actitud contemplativa en el trabajo plástico, cosa no común en los artistas occidentales contemporáneos que son predominantemente activos. Supongo que habrás frecuentado los libros que hablan de los do japoneses, es decir los diversos caminos laicos hacia la realización interior, entre los cuales son muy importantes los estéticos, como el camino de las flores, el de la caligrafía, el de la pintura" [FALta Referencia Y BIBLIOGRAFÍA]. 
interior y el espacio exterior se hacen uno en esa composición verbal que Padeletti va construyendo con atención y paciencia.

Y aquí quiero trazar una analogía entre el zen, el arte del té y los poemas del rosarino. De acuerdo con Daisetz T. Suzuki, "un elemento común al zen y el arte del té es la constante atención que ambos presentan a la sencillez. La eliminación de lo innecesario es realizada por el zen en su intuitiva captación de la realidad final [...] El arte del té es la expresión estética de una simplicidad original" (1996: 182). Algo muy similar sucede en la poética padelettiana:

POCAS COSAS

y sentido común

y la jarra de loza, grácil,

con el ramo

resplandeciente.

La difícil

extracción del sentido

es simple:

el acto claro

en el momento claro

y pocas cosas-

verde

sobre blanco (Padeletti, 2018a: 129).

Este poema puede ser tomado como un "arte poética": construir el poema con lo mínimo necesario para originar el vacío. La concepción que está detrás es similar a la de la habitación del té, que supone un espacio vacío y a la vez multivalente. El vacío se 
crea a partir de la forma. La hoja de papel en blanco anota Padeletti, no es más que el papel; solo cubriéndola de signos se crea en ella el vacío. Lo mismo sucede en la habitación del té o la "Casa del Vacío", como también la llama Okakura Kakuzo, siguiendo uno de los significados que se desprenden de los ideogramas originales de la palabra Sukiya. El término "implica [señala Kakuzo] la idea de una necesidad continua de cambiar los motivos decorativos. La habitación está vacía, excepto por lo que se puede colocar en ella temporalmente" (Kazuko, 2011: 65). ${ }^{2}$ El maestro del té introduce en la habitación vacía algunos objetos para resaltar su belleza y propiciar el estado de concentración-contemplación de los participantes. Los principios que debe respetar para propiciar la atmósfera adecuada son, de acuerdo con Suzuki: "armonía (wa), reverencia ( $k e i)$, pureza (sei) y tranquilidad (jaku)". De los cuatro, es la tranquilidad el fundamento, es "el principio que gobierna el arte del té, donde falta, el arte perdería por completo su significado" (Suzuki, 1996: 204). La vida de un poema para Padeletti pende de principios similares y también el poeta sitúa a la tranquilidad en el centro:

Ahora sé que una obra tiene vida o no la tiene y, si la tiene [...] lo que importa es qué clase de vida. La que a mí más me interesa y la que más necesita, creo, el perturbado hombre de hoy es tranquila, serena, simple, silenciosa, profunda, transparente como agua en reposo; proviene de la experiencia contemplativa y reconduce nuevamente a ella (1999b: 152).

${ }^{2}$ Para Padeletti decorar es "honrar el espacio": "Yo uso la palabra decoración en ese sentido y no en el peyorativo que tiene modernamente. Decorar el plano es, etimológicamente, honrarlo como plano, cohonestarlo, en el sentido del 'cohonestare' latino, que significa realzar, embellecer. Lo cual implica al mismo tiempo otorgarle un significado, un sentido, porque la belleza es siempre epifanía y en última instancia Presencia” (1994a: 21). 
Tanto la habitación del té como el poema generan un espacio armónico, sereno, en el que el hombre puede olvidase de sí para regresar a su "sí mismo", y abrir sus sentidos y su mente. Proporcionan, en suma, un espacio para la contemplación y el autodespertar.

Entrar y sentarse es la clave de ambos espacios. C. E Feiling se detiene en un procedimiento que se repite con frecuencia en los poemas de Padeletti: "[el poeta] funde el título con el poema: el título es el primer verso, pero su cuerpo tipográfico distinto, y el espacio que el lector debe recorrer con la vista para llegar al aparente primer verso logran, en combinación, un efecto curioso. Es como si uno descubriera que ya estaba leyendo el poema antes de comenzar" (Padeletti, 1999c: 276). Los poemas de Padeletti invitan al lector a entrar en ellos, así como el maestro del té invita a su ceremonia a un pequeño grupo de participantes. La arquitectura del Sukiya consiste en la habitación del té, en la que se reúnen los pocos invitados; una antecámara, en la que se preparan y lavan los utensilios para tomar el té; "un pórtico (machiai), en el que los invitados esperan hasta que les hacen entrar, y un sendero (el rôji) que comunica el machiai con la habitación del té" (Kazuko, 2011: 54). Haciendo una analogía con los poemas, que podría resultar sugerente, el pórtico o título, en letras mayúsculas, separado del resto del poema, aunque ya parte primordial de él, invita al ojo del lector a transitar por el sendero (espacio vacío que Padeletti introduce) hasta situarse-sentarse tranquilamente y contemplar los resquicios que se abren entre las palabras-cosas del poema. En el poema anteriormente citado, lo que se ve es un jarrón (blanco) con un ramo (verde) resplandeciente. Podría ser, si seguimos la analogía con la habitación del té, un arreglo floral (ikebana) que se posa en una de las paredes de la habitación (tokonoma) para disfrute de quien lo observa. Lo simple del arreglo esconde la complejidad y 
profundidad de su sentido. ${ }^{3}$ Dentro de las leyes de composición formuladas por diversos maestros, todo arreglo floral debe incorporar "al Principio Dominante (el Cielo), al Principio Subordinado (la Tierra) y al Principio Conciliador (el hombre)" (Kazuko, 2011: 99). Tanto el Ikebana como la habitación del té en el que este se encuentra suponen una "geometría moral", puesto que definen el sentido de nuestra proporción con respecto al universo (Kazuko, 2011: 6). Esa "geometría moral" no le es extrańa a Padeletti, ya que para él crear un objeto plástico o de palabras supone una "contemplación activa" en la que la formaadquiere un papel primordial. ${ }^{4}$ En esa misma línea Monteleone señala: "[...] la poesía de Padeletti es una forma moral. En sus poemas reaparecen los vocablos que aluden a esa especie de impersonalidad de la conciencia: lo desistido, lo desasido, la desafección, lo desentendido. Pero esa conciencia no desiste al punto de olvidar las formas concretas del mundo, antes bien se concentra en ellas, se activa en ellas, transige entre ellas y las formas del poema" (Padeletti, 2018b: 1113).

${ }^{3}$ El poema de Padeletti está dedicado a la memoria de Juan Grela, pintor argentino y maestro del poeta. Por lo que este bien podría aludir a una de las pinturas de Grela, quien gustaba de hacer jarrones y flores. Como sea, me parece que la analogía con la habitación del té se sostiene, cuanto más si pensamos que en el tokonoma debía de haber siempre un rollo de pintura (kakemono) colgando para ser contemplado por los participantes.

${ }^{4}$ Los poemas de Padeletti presentan un cuidado particular en la forma. Guillermo Saavedra seńala algunos de los recursos que permiten la eficacia y coherencia de la misma: "la disposición del poema (único) en la página, suerte de composición o puesta en escena que parece mimar la dirección -lateral, vertical o sinuosa- del pensamiento; las citas más o menos embozadas; la aparición irregular de rimas internas y finales, a veces deliberadamente homofónicas [...] la utilización casi constante de metros clásicos de la poesía española como el endecasílabo y el heptasílabo. En una inestabilidad aparente, sometidos a enmascaramientos, rupturas engañosas, intercalaciones de otros metros (tetrasílabos y pentasílabos), estos dos patrones métricos son los pulmones rítmicos de la poesía de Padeletti” (Apud. Padeletti, 1999d: 290). 
El hombre es el conciliador, su conciencia transige, pero no es el centro; es un elemento más dentro de la habitación y también uno más dentro del poema. No se yergue sobre las formas del mundo o las formas creadas, se sienta tranquilamente y se sitúa entre ellas:

HUMORES DEL JARDÍN

abrumado de olor.

No hay corola de flor

que no perfume el fin.

Pero sigue la rueda y la semilla se parapeta para un nuevo lance.

Después de oscuro trance

se engalana de nuevo el florecer.

Yo, sentado en la silla

de mecer,

me preparo de nuevo a renacer (Padeletti, 2018a: 507).

No me parece casual que en los poemas del rosarino abunden las flores, para el Maestro Prajñătara, “la apertura de las flores es la existencia del mundo', sugiriendo que el mundo real en sí mismo es justamente la aparición de los fenómenos" (Dogen, 2016a: 270). Es el olor de las flores el que en el poema lleva al "yo" a florecer. "El yo florece en su desprendimiento de sí mismo" (Ueda, 2005: 103), al que se alude en ese renacer que supone que antes hubo un morir. Se muere a la cerrazón del "yo" y se renace a una apertura de este. "Al ser partícipe del mero las-flores-florecen, el hombre, por la fuerza presencial de esa percepción, es reducido a la nada y despertado de nuevo a la vida en tanto en cuanto florece 
junto con las flores. En ese instante tiene lugar en el hombre el acontecimiento de la muerte- resurrección" (Ueda, 2005: 30), (el yo no-yo) o el autodespertar, muy distinto al autoconocimiento que implica un proceso racional, mientras que el primero se sostiene en la repentina intuición de la vida. El autodespertar procede de la claridad y de la transparencia: "el acto claro / en el momento claro". Es precisamente la manifestación de lo ordinario y no de lo extraordinario lo que lo produce: las flores florecen.

El Maestro Eihei Dogen en el Shobogenzo cita el poema del Maestro Zen Raiun Shingon:

Durante treinta años, un viajero buscando una espada ¿cuántas veces han caído las hojas y germinado los brotes? Tras una mirada a los melocotones en flor, he llegado directamente al presente y no tengo más dudas (2016: 147).

El poema de Padeletti, al igual que este bello poema de Shingon, "expresa el mundo o las cosas en su ser así, que brilla fuera de la intervención humana” (Han, 2015: 106). Lo que se manifiesta es lo que se manifiesta, no hay nada que esconder. En ese "estar en el presente" todo es claridad y apertura:

Hay una soledad,

y con el canto

del gallo se resuelve,

y se abren para ti las Estaciones (Padeletti, 2018a: 44).

La jarra de loza con el ramo resplandeciente, el olor de las flores que florecen o el canto del gallo, son el pivote para que surja la apertura y el despertar. Padeletti, como el monje zen, "habita el 
mundo de la apariencia [...] se mantiene en una inmanencia con muchas formas" (Han, 2015: 44). Suzuki, al reflexionar sobre el conocido haiku de Basho sugiere: 5

[La] comprensión intuitiva de la Realidad no surge cuando un mundo de Vacuidad es asumido fuera de nuestro cotidiano mundo de los sentidos; pues estos dos mundos, sensible y suprasensible, no están separados, sino que son uno. Por tanto, el poeta penetra con su visión al interior del inconsciente no a través de la serenidad del viejo estanque sino a través del sonido provocado por el salto de la rana. Sin el sonido no hay visión por parte de Basho del inconsciente en el que descansa la fuente de las actividades creativas de la que todos los verdaderos artistas sacan su inspiración (1996: 163).

Los fenómenos del mundo y el vacío son uno, o como reza la paradoja budista en el Sutra HannyaShin: "lo sensible (forma) igual a lo vacío y lo vacío igual a lo sensible (forma)" (Shinichi, 2011: 87). En palabras de Hugo Padeletti: "El vacío expresado [...] por la elipse en poesía [...] sólo puede ser creado y comprendido por medio de las formas estéticas que sabiamente lo pueblan" (1994a: 15). La omisión y a la vez la precisión que supone la elipsis son un punto clave en el arte poético que despliega el poeta; en ese no explicar ni justificar, en ese no decir, se sostiene la realización intuitiva de la obra, que supone detenerse y mirar: "VISIÓN, ATENCIÓN Y sOsIEgo / desde el principio / y disponibilidad para ver / desde otro sesgo. // El polvo que al sol flota y destella / revela la secreta incandescencia / de lo grueso" (Padeletti, 2018a: 950). O

5 "El viejo estanque, jah! / salta una rana: / ¡el sonido de agua!" (Apud. Suzuki, 1996: 154). 
bien, desestabiliza a la mente para en lo posible detener el pensamiento conceptual:

MESA REDONDA

- Si el freudismo consiste

en convertirte en tripas,

corazón, y el marxismo

encoge con el uso,

¿entonces qué?

—El progreso

no puede operarse.

Terció el pobre: - Mejor

desnudo que encogido.

Y el panzudo: - Hacer

de tripas corazón.

A la larga - 'non multa

sed multum'-

habló el toro:

dijo MU (Padeletti, 2018a: 208).

Con humor y elegancia, Padeletti cierra su poema con el koan Mu que significa "nada". ${ }^{6}$ Enfrentando el discurso freudiano y marxista a la nada budista. El koan $M u$, conocido como "la primera barrera”, abre el Mumonkan (La barrera si puertas), libro que está compuesto por cuarenta y ocho koanes. Este koan se asocia al

${ }^{6}$ El Maestro Dogen consideraba varios significados del carácter $m u$, "estado real, carencia de posesión y ausencia” (2016b: 72). 
maestro Zhaozhou Congshen (conocido también como el Patriarca Joshu) de la dinastía Tang:

Un monje le preguntó a Zhaozhou: ¿Tiene un perro naturaleza búdica, o no?

Zhaozhou dijo: $\mathrm{Mu}$ (Ueda, 2005: 45).

El comentario del Maestro Zen Wu-men (Mumon), compilador de la colección, es el siguiente: "En la práctica del Zen debes atravesar la puerta de la barrera impuesta por los patriarcas. Para realizar esta cosa maravillosa llamada iluminación debes romper con todo pensamiento (discriminativo)" (Kapleau, 1988: 98). El koan, central en la práctica de la escuela Rinzai, tiene como objeto desestabilizar el pensamiento racional y abrir la mente del practicante para que este vaya más allá del pensamiento ordinario. El maestro no pretende una respuesta o solución de este, lo que busca es que el discípulo investigue el koan con toda su atención y que llegue a ser uno con él. De ahí la eficacia del final del poema MESA REDONDA: el toro se hace uno con MU. MU es en el poema un koan, pero también el mugido del toro (la voz animal); es asimismo una onomatopeya que rompe con el pensamiento discursivo y especulativo que se había puesto sobre la mesa. MESA REDONDA supone un diálogo entre las ideas, las opiniones y las necesidades, en el que parece no haber salida. En ese enfrentamiento de ideas se inscribe el lema latino de Plinio el joven, que aconseja: "non multa/sed multum" (no muchas cosas, sino mucho), es decir, mejor profundizar en el conocimiento en vez de acumularlo. En Plinio aparece la chispa de la sabiduría, sin embargo, $M u$ está aún más allá o más acá de esta: "Mu se mantiene fríamente por encima tanto del intelecto como de la imaginación" (Kapleau, 1988: 91): no es ni exaltado ni moderado; ni sabio ni tonto, es más bien un acontecimiento. MU es por lo mismo, cierre y apertura: el toro no participa de las 
ataduras de la ilusión, está libre de ellas y es uno con el mundo, su mugido irrumpe como un elemento liberador. El acontecimiento MU introduce una resquebrajadura en el universo lingüístico; onomatopeya, voz animal o koan, deja a los participantes de la discusión sin palabras y por lo tanto los enfrenta al silencio o al puro sonido. Padeletti, en suma, de manera lúcida y juguetona actualiza un mondo zen, insertándolo en una discusión netamente occidental y llevándolo, a partir de una aguda apropiación, a su presente. No está de más hacer notar que ya en el lema latino: "non multa /sed multum" está resonando $m u$, tres veces y en espejo: MU, pareciera deslizarse de forma natural y espontánea al final poema.

Padeletti no se piensa a sí mismo como un maestro zen, se sabe artista y conoce en ese sentido su camino: "Los caminos del arte no son los de la Unidad Última sino los de la infinita variedad de las epifanías posibles. En el arte gozamos, sí, virtualmente, de la Unidad, pero se trata de la 'unidad en la variedad', en la ilimitada variedad de lo sensible" (1994b: 48). El zen, escribe también, "no es una moda, sino una tradición prácticamente intemporal y su posible influencia está viva y abierta en cada momento, sólo hay que pasar la antorcha". Y después agrega: "Hay un zen hindú, un zen chino, un zen japonés y un zen universal. Este último existe y existió siempre en occidente. Se lo puede encontrar -en semillaen los clásicos griegos y latinos, en la Biblia, en los místicos, poetas, filósofos y hasta científicos de diversas épocas". Es precisamente en este zen intemporal y universal en el que converge la obra padelettiana, y son algunos trazos del mismo los que en este trabajo se han intentado seguir. 


\section{Bibliografía}

Deshimaru, Taisen, 2000, La práctica del zen, Nieves Semblancat y Pere Rovira (trad.), Editorial Kairos, Barcelona.

Dogen, Eihei, 2016a, Shobogenzo (Tesoro del Verdadero Ojo del Dharma), vol. 1, Pedro Piquero y Gudo Wafu Nishijima (trad.), Editorial Sirio, España.

, 2016b, Shobogenzo (Tesoro del Verdadero Ojo del Dharma), vol. 2, Pedro Piquero y Gudo Wafu Nishijima (trad.), Editorial Sirio, España.

Han, Byung-Chul, 2015, Filosofía del Budismo Zen, Raúl Gabás (trad.), Herder, Barcelona.

Kapleau, Roshi Philip, 1988, Los tres pilares del Zen. Enseñanza, práctica, iluminación, Marta Carpio Carreón (trad.), Árbol Editorial, México.

Kazuko, Okakura, 2011, El libro del té, Esteve Serra (trad.), José J. de Olañeta Editor, Barcelona.

Padeletti, Hugo, 1994a, "Zen y pintura pura (a propósito de la muestra de Jorge Abot en la OEA", en Textos ocasionales, Bajo la luna nueva, Rosario, Buenos Aires.

, 1994b, "La pintura de Mariano Cornejo (contorno, dintorno y entorno de una experiencia)", en Textos ocasionales, Bajo la luna nueva, Rosario, Buenos Aires.

, 1999a, "Para una metafísica del ojo y otras indiscreciones", en La atención. Obra reunida. Poemas verbales-poemas plásticos, t. I, Universidad Nacional del Litoral, Santa Fe, Argentina.

, 1999b, "Historia personal y consideraciones al margen" en La atención. Obra reunida. Poemas verbales-poemas plásticos, t. I, Universidad Nacional del Litoral, Santa Fe, Argentina. 
, 1999c, "Poemas 1960/1980" en La atención. Obra reunida. Poemas verbales-poemas plásticos, t. III, Universidad Nacional del Litoral, Santa Fe, Argentina.

, 1999d, "La voz de la experiencia", en La atención. Obra reunida. Poemas verbales-poemas plásticos, t. III, Universidad Nacional del Litoral, Santa Fe, Argentina.

, 2004, Dibujos y poemas 1950-1965, Editorial Áncora, Buenos Aires.

, 2018a, Poemas Completos, Adriana Hidalgo Editora, Argentina / España.

, 2018b, "Ahora es ahora es ahora. La poesía de Hugo Padeletti”, en Poemas Completos, Adriana Hidalgo Editora, Argentina / España.

Shinichi, Hisamatsu, 2011, Los Cinco Rangos del Maestro Zen Tosan, Ana María Schlüter Rodés (Kiun An) (trad.), Herder, Barcelona.

Suzuki T., Daisetz, 1996, El zen y la cultura japonesa, María Tabuyo y Agustín López (trad.), Editorial Paidós, España.

Ueda, Shizeteru, 2005, Zen y filosofía, Raquel Bouso García e Illiana Giner Comín (trad.), Herder Editorial, Barcelona.

Verdejo-Padeletti, Correspondencia en correos electrónicos. Archivo personal de Luis Verdejo. 